\title{
Protective effects of Wenxin Keli against cardiac arrhythmias (Review)
}

\author{
SHRISTI DAHAL, MENGQI GONG, SHAOHUA GUO, GARY TSE and TONG LIU \\ Tianjin Key Laboratory of Ionic-Molecular Function of Cardiovascular Disease, Department of Cardiology, \\ Tianjin Institute of Cardiology, Second Hospital of Tianjin Medical University, Tianjin 300211, P.R. China
}

Received December 19, 2019; Accepted March 30, 2020

DOI: $10.3892 /$ wasj.2020.43

\begin{abstract}
Cardiac arrhythmias represent a significant problem globally. Combined traditional Chinese and Western medicine is being integrated into current therapeutic trials in continued efforts to suppress arrhythmias that are predicted to escalate in the face of increasing life expectancy and medical advances. This review highlights regulatory mechanisms and major target sites of a patented, traditional Chinese drug, Wenxin Keli (WXKL). It describes the roles of individual components of WXKL via multiple pathways in interrupting reentry, reducing automaticity and preventing arrhythmogenic substrates. It further elucidates the effects of WXKL on atrial and ventricular electrophysiology, highlighting its weaker effects on rapid sodium curent (INa), as well as its multi-channel block, apart from atrial selectivity. In addition, the present review article incorporates diverse actions of WXKL in the reduction of reactive oxygen species levels, CX43 regulation, calcium handling, $\mathrm{Ca}^{2+} /$ calmodulin-dependent protein kinase II (CaMKII) modulation and neurohumoral system regulation. Apart from its role in managing angina and heart failure shown in experimental and clinical models, the aforementioned effects enable WXKL to maintain sinus rhythm and prevent cardiac arrhythmias and sudden cardiac death associated with multiple comorbidities. Based on the results from various studies, it has minimal side-effects and is less proarrhythmic with an efficacy that matches that of western drugs. Varying effects of WXKL on multiple ion channels, intercellular conduction, and second messenger and redox systems have opened novel doors in managing arrhythmias.
\end{abstract}

Correspondence to: Professor Tong Liu, Tianjin Key Laboratory of Ionic-Molecular Function of Cardiovascular Disease, Department of Cardiology, Tianjin Institute of Cardiology, Second Hospital of Tianjin Medical University, Tianjin 300211, P.R. China

E-mail: liutongdoc@126.com

Key words: Wenxin Keli, arrhythmias, Nardostachys jatamansi DC (Gansong), Radix Notoginseng (Sanqi), Succinum (Hupo), Polygonatum sibiricum (Huangjing), Codonopsis pilosula (Dangshen)

\section{Contents}

\author{
1. Introduction \\ 2. Roles of individual components of WXKL \\ 3. Effects of WXKL on atrial electrophysiology \\ 4. Effects of WXKL on ventricular electrophysiology \\ 5 . Reactive oxygen species (ROS) reduction \\ 6. Gap junction modulation \\ 7. Calcium handling \\ 8. CaMKII signaling \\ 9. Autonomic nervous system regulation \\ 10. Conclusion
}

\section{Introduction}

Cardiac arrhythmias pose a significant global burden, resulting in cerebrovascular accidents, myocardial infarction and sudden cardiac death (SCD) (1). The worldwide prevalence of atrial fibrillation (AF), characterized by uncoordinated atrial activation with the consequent deterioration of atrial mechanical function, is 37,574 million cases $(0.51 \%$ of worldwide population) (2). The prevalence of AF is escalating in middle socio-demographic index countries, and its absolute burden is projected to increase by $>60 \%$ in 2050 , being one of the largest epidemics (2). The prevalence of AF is approximately $0.77 \%$ in China, with the mean age of patients being $52.5 \pm 22.4$ years (3), with a lifetime risk of $1 / 5$ among Chinese adults based on a medical insurance database involving 10 million in Southwest China (4). Premature ventricular contractions (PVCs) manifest from being asymptomatic to the development of cardiomyopathy, heart failure, sustained ventricular tachycardia (VT) or even total mortality. In Europe and North America, 50-100 unexpected SCDs occur per 100,000 population annually, half of these events owing to VT or ventricular fibrillation (VF) (5).

Although classic Western anti-arrhythmics that tend to interact with a specific or single target mostly via inhibition possess potent pharmacologic activity, they portend risks of breaking intracellular stability and have been reported to be arrhythmogenic (6). Consequently, the development of safe and effective regimens remains a major focus of contemporary cardiovascular research. With globalization, an integration of the traditional Chinese and Western medical practices has 
begun in multiple medical centers (7). In recent years, network pharmacology is an effective approach which can be used to address the association between multiple TCM components and the synergistic effect of drugs on a series of targets. Modern Chinese herbal medicines manufactured according to traditional theory, play a significant role in the treatment of cardiac arrhythmias (8).

Wenxin Keli (WXKL) is a first-state sanctioned Traditional Chinese Medicine (TCM)-based anti-arrhythmic drug (8). It was officially marketed in 1995 as it received new certification issued by the Ministry of Health of the People's Republic of China (9). In China, it possesses a licensable indication for PVCs, which was incorporated in the 2009 revision of the National Reimbursement Drug List (10). As a sole anti-arrhythmic herbal extract in the 2010 revision of Chinese Pharmacopoeia, almost 5 million Asians are currently being administered WXKL for the treatment of ventricular arrhythmias (11). Wenxin Keli is composed of 5 constituents: Nardostachys jatamansi DC (Gansong), Radix Notoginseng (Sanqi), Succinum (Hupo), Polygonatum sibricum (Huangjing) and Codonopsis pilosula (Dangshen) (4,6,12-14). Dangshen, the 'monarch' in the regimen, characterized by a sweet flavor, boosts 'qi' (nutrients), tranquilizes the mind and relieves palpitations. Huangjing, the 'minister' herb, enhances the effects of Dangshen, and also possesses a sweet flavor and fortifies the spleen 'qi' to nourish the heart and lungs. The remaining 3 ingredients are assistant herbs. The bitter-flavored Sanqi removes blood stasis, relieves pain and tonifies the system. Hupo, neutral and sweet, pacifies the liver and tranquilizes the mind. Gansong is warm and sweet, relieves depression and regulates the liver and spleen 'qi'. Its indications include palpitations, dyspnea, fatigue, dizziness, chest tightness and pain, and insomnia to improve the quality of life $(9,15)$.

In a rat model of ischemia-reperfusion (I/R) injury, pre-treatment with WXKL was shown to effectively improve cardiac hemodynamics, accelerate coronary blood flow, enhance myocardial contractility, and reduce the occurrence of arrhythmias $(16,17)$. WXKL reportedly increases coronary blood flow, reduces myocardial oxygen consumption, enhances myocardial compliance, improves myocardial hypoxia tolerance, relieves anterior and posterior cardiac loading, and reduces arrhythmic occurrence (16). The aim of the present review article was to review the effects of WXKL on cardiac electrophysiology and how these can prevent cardiac arrhythmias.

\section{Roles of individual components of WXKL}

Nardostachys jatamansi DC (Gansong). Nardostachys chinensis batal extract $(\mathrm{NcBe})$ at $10 \mathrm{~g} / 1$ has been shown to inhibit both peak INa and transient outward potassium current (Ito) in rat ventricular myocytes. These actions were associated with the suppression of acetylcholine-induced AF or atrial flutter, likely by reducing both triggered activity and reentry $(18,19)$. Additionally, it has been shown to inhibit L-type calcium currents (ICaL) and shift the current-voltage association upward without changing its activation or reversal potential (9). Calaxin, one of its components, has been predicted to be a CaV1.2 channel blocker (6). The dried roots of $\mathrm{NcBe}$ contain sesquiterpenoids, which can lower the incidence of ventricular arrhythmias and improve $\mathrm{Cx} 43$ redistribution in rats with hyper-acute myocardial infarction (MI) (20). This is probably due to the lowering of the heterogeneity of conduction (21). Moreover, $\mathrm{NcBe}$ contains valerian ketones that can reduce myocardial cell automaticity, extend the atrial action potential (AP) of the conduction system time and interrupt reentry (15). The volatile oil of Nardostachys can inhibit oxidative stress-induced cell death via reactive oxygen species scavenging and Akt activation in $\mathrm{H} 9 \mathrm{c} 2$ cardiomyocytes (22). Since increased oxidative stress is associated with arrhythmogenesis, this may act to suppress arrhythmias (1). Nardosinone, one of the major extracts from Nardostachys, has been shown to prevent angiotensin II (AngII)-induced cardiac hypertrophy in embryonic rat hearts, particularly at a concentration of $\geq 100 \mu \mathrm{mol} / 1$ by inhibiting the phosphatidylinositol 3-kinase (PI3K)/Akt and MEK/ERK pathways (23).

Radix Notoginseng (Sanqi). Notoginseng has been reported to antagonize $\mathrm{Na}^{+} / \mathrm{K}^{+}$-ATPase activity (24) and the effect of acetylcholine on various human organs (18). This may reduce sinus node and ectopic pacemaker activity, and improve microcirculation (15). It exerts multiple cardio-protective effects, including pro-angiogenesis, anti-apoptotic, inflammatory cascade repression and endothelium-dependent vasodilation (22). It protects hypoxia-reoxygenation induced $\mathrm{H} 9 \mathrm{c} 2$ cardiomyocyte injury at the dose of $25 \mu \mathrm{g} / \mathrm{ml}$ associated with apoptosis via the phosphatidylinositol 3-kinase (PI3K)/AKT and mitogen-activated protein kinases (MAPKs) pathways (25). Pre-treatment with ginsenoside, an active ingredient of Notoginseng, has been shown to protect rat cardiomyocytes from oxidative injury by the expression of respiratory chain complexes via the RhoA/ROCK signaling pathway (26) and through an increase in the activity of endogenous antioxidants and suppression of intracellular $\mathrm{Ca}^{2+}(27)$. According to a systematic review and meta-analysis of 18 randomized controlled trials (RCTs), Notoginseng was shown to reduce cardiovascular events, such as acute myocardial infarction (AMI), severe arrhythmia, heart failure and intractable angina pectoris (28). Through the integration of the gene interaction network, the role of Notoginseng in the treatment of coronary heart disease has been shown to involve the cytokine-cytokine receptor interaction pathway (29). It has also been shown to mitigate aging-induced cardiomyocyte apoptosis possibly by increasing oxidative stress, and mitochondrial function-related signaling pathways, such as the manganese superoxide dismutase (MnSOD) signaling pathway, and mitochondrial-related signaling molecules, such as peroxisome proliferator-activated receptor-gamma coactivator (PGC)-1 $\alpha$, light chain (LC) $3 \beta$ and Beclin-1 (30).

Succinum (Hupo). No effect of succinum on cardiac physiology has been found to date, at least to the best of our knowledge.

Polygonatum sibiricum (Huangjing). Polygonatum sibiricum exhibits lipid-lowering and anti-atherosclerotic effects and tends to reduce blood pressure $(15,20)$. Apart from increasing $\mathrm{Na}^{+}-\mathrm{K}^{+}$-ATP and $\mathrm{Ca}^{2+}$-ATP activity in mouse brain cells, Huanjing enhances anti-aging effects by reducing lipid peroxide and B-type monoamine oxidase levels. Its anti-atherosclerotic effect may be associated with the reduction of foam cells and hypolipidemic activity (31). In addition, its protective effects 


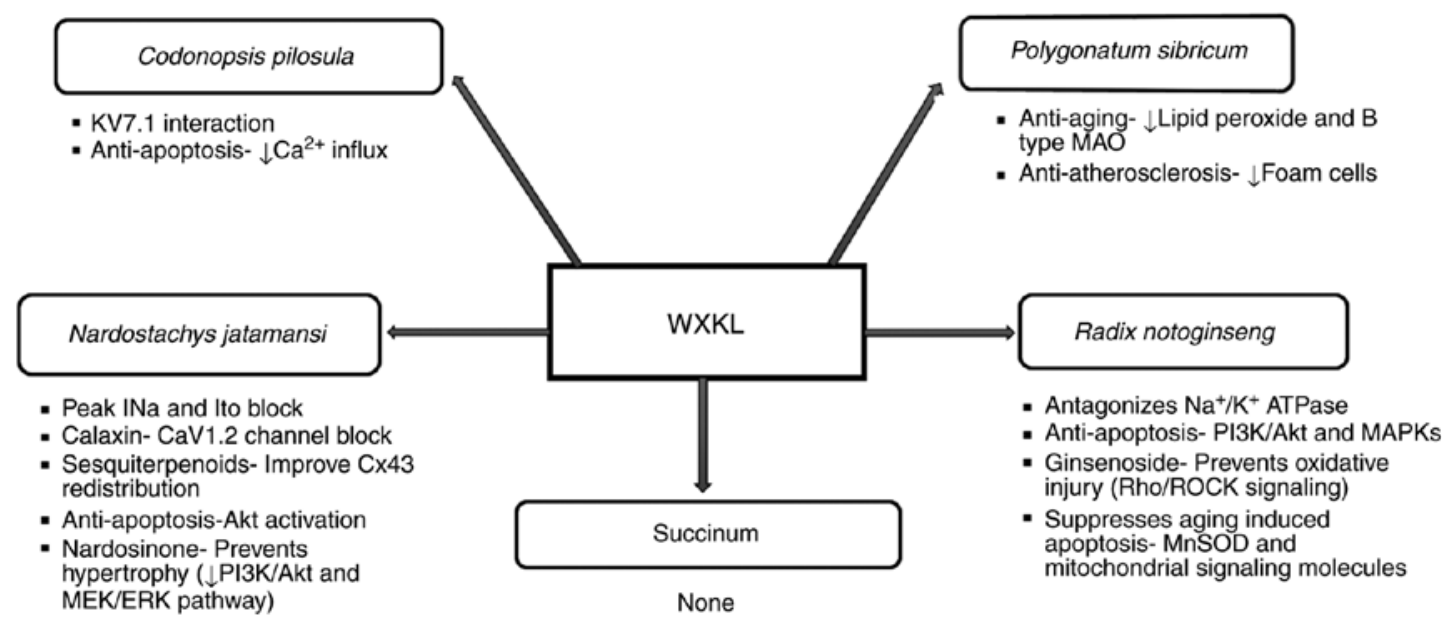

Figure 1. Diverse roles of individual components of Wenxin Keli. AKT, protein kinase B; CaV1.2, L-type voltage-dependent calcium channel 1.2; ERK, extracellular regulated protein kinases; INa, fast sodium curent; Ito, transient outward potassium current; KV7.1, voltage-activated $\mathrm{K}^{+}$channel 7.1; MAO, monoamine oxidase; MAPKs, mitogen-activated protein kinases; MEK, mitogen extracellular signal-regulated kinase; Mn-SOD, manganese superoxide dismutase; PI3K, phosphatidylinositol 3-kinase; Rho/ROCK, ras homolog gene/a Rho-associated coiled coil-forming proyein kinase; WXKL, Wenxin Keli.

on adriamycin-induced acute heart failure in rats may be related to its anti-inflammatory effects and to the inhibition of cardiomyocyte apoptosis (32).

Codonopsis pilosula (Dangshen). The component codonopsine is predicted to interact with KV7.1 that is significant in cardiac repolarization phases 2 and 3 (6). It contains inulin and amino acids, and exerts anti-platelet aggregation and enhances immunity (15). It attenuates calcium influx and apoptosis induced by angiotensin II in $\mathrm{H} 9 \mathrm{c} 2$ cardiomyoblasts (22). It has been reported to induce phosphodiesterase inhibition, leading to an increase in cAMP levels in rat myocytes (24). Codonopsis has been shown to reverse the AngII plus Leu27 insulin growth factor II (IGFII)-induced caspase-3 activity and the apoptosis of $\mathrm{H} 9 \mathrm{c} 2$ cardiomyoblasts (33) by reducing $\mathrm{Ca}^{2+}$ influx and mitochondrial outer membrane permeability (34) (Fig. 1).

\section{Effects of WXKL on atrial electrophysiology}

Sodium channel. The major atrial-ventricular $\mathrm{Na}$ channel disparities are a more negative atrial INa steady-state inactivation and a slower recovery from inactivation (35). Peak INa is responsible for phase 0 of the action potential. The density of peak INa is significantly greater in atria than in ventricles, highest in Purkinje cells (36).

The anti-AF effects of peak INa blockers are due largely to the rate-dependent reduction of excitability, the prolongation of the effective refractory period (ERP), and to the conduction block in a critical part of reentrant circuit. The reduction of peak INa can also significantly decrease intracellular sodium (Nai) and, thus, intracellular calcium (Cai), which may suppress Cai-mediated triggered activity (37). Severe structural remodeling, resulting in a small number of connections between the subendocardial and subepicardial layers of the atria, can prevent termination of AF by Na-channel block (38).

While the atrial selectivity of other INa blockers encompasses a preferential prolongation of action potential duration (APD), WXKL at $5 \mathrm{~g} / 1$ abbreviates $\mathrm{APD}_{90}$ predominantly in the atria (18). It shifts the steady-state availability to more negative potentials in atrial than in ventricular cells in a dose-dependent manner (39). Such differing atrial and ventricular effects may possibly be due to different ratios of alpha and beta subunits. WXKL binds to the inactivated state of peak $\mathrm{I}_{\mathrm{Na}}$ and dissociates rapidly (39). Following ganglionated plexi (GP) ablation, it has been shown to significantly attenuate the levels of atrial natriuretic peptide (ANP), tumor necrosis factor (TNF)- $\alpha$ and interleukin (IL)-6 within 10 weeks of oral administration at $0.25 \mathrm{~g} / \mathrm{kg} / \mathrm{day}$, suggesting its anti-inflammatory effects (40).

The combination of $18 \mathrm{~g} 3$ times daily of WXKL and amiodarone has been shown to be safe and superior to amiodarone monotherapy in the chemical conversion of recent-onset AF, exhibiting a markedly shortened time in sinus rhythm restoration (41). In another study, the same dose of WXKL and $80 \mathrm{mg}$ sotalol twice a day was shown to exhibit a similar efficacy to assist sinus rhythm reversion from hyperthyroidism-caused paroxysmal atrial fibrillation (PAF) (42).

\section{Effects of WXKL on ventricular electrophysiology}

Sodium channel. Late sodium channel (INaL) maintains phases 2 and 3 of cardiac AP. The rate-dependent reduction of INaL contributes to rate-dependent APD abbreviation (43). The contribution of INaL is more significant during prolonged APD and bradycardia. VT/VF most commonly occur under the conditions of prolonged APD (44). The INaL inactivation occurs in several hundreds of milliseconds and persists throughout the AP plateau.

INaL suppresses VAs by reduction of repolarization heterogeneity and intracellular $\mathrm{Ca} 2+$ secondary to the decrease of late INa mediated Nai (37). Increased intracellular Ca2+ alters repolarization such as T wave alternans and causes DADs (45). Its distribution is spatially heterogeneous in the ventricles (46). INaL blockers such as ranolazine effectively prevent or shorten rapid activation-induced VT and VF in non-diseased ventricles (47).

In addition to its atrial selectivity, $5 \mathrm{~g} / 1 \mathrm{WXKL}$ has only a mild effect in reducing ventricular rapid INa. It binds very rapidly to the inactivated sodium channel and dissociates 
rapidly from the closed state (39). WXKL suppresses ventricular early afterdepolarizations (EADs), delayed afterdepolarizations (DADs) and triggered activities (TAs) by the selective inhibition of late INa. The IC50 of the late INa by WXKL was approximately 3 -fold higher than that of the rapid INa in the rabbit left ventricle. In addition, its weaker influence on rapid INa with little use-dependence unlike classic INa blockers renders it less proarrhythmic (48). WXKL at both 1 and $5 \mathrm{~g} / 1$ exhibits anti-arrhythmic effects on Purkinje cells of rabbit hearts via INaL inhibition by the shortening of APD and attenuating ATX-II and ISO-induced EADs and DADs. The average magnitude of INaL in these cells exceeded by 3 -fold compared to that in ventricular cells (12).

Potassium channel. Ito is the transient outward current resulting from $\mathrm{K}^{+}$efflux. It is a key regulator of phase one action potential repolarization. Ito varies in early-onset lone AF, Brugada syndrome and idiopathic VF (49). It is regulated by cold-inducible RNA-binding protein (50). Computational modeling predicts that in human ventricle, small decreases in Ito slightly increase APD, while large increases can shunt the $\mathrm{AP}$ and cause rapid repolarization (51).

The majority of SCD occurrences in patients with heart failure (HF) is likely secondary to VAs. Heart failure with preserved ejection fraction $(\mathrm{HFpEF})$ increases the inducibility of VA as a result of delayed repolarization and multiple reentry circuits demonstrated by prolonged repolarization and Ito downregulation, contributing to arrhythmogenesis (52).

WXKL at the dose of $8 \mathrm{~g} / \mathrm{kg}$ daily has been shown to prevent VAs in vivo following the long-term administration in a rat model of MI associated with inhibition of $\mathrm{ICaL}$ and Ito. It accelerates Ito inactivation without changing the activation process or the recovery of its inactivation (8). The mutual mechanism in HF and arrhythmia includes inflammation, oxidative stress and microRNA regulation. Based on a systematic review and meta-analysis on 13 studies published in Chinese, WXKL plus amiodarone combination group was shown to be superior in total effective rate, heart function and reduction in PVCs compared to amiodarone alone. WXKL downregulates genes associated with inflammation, neurohumoral system and apoptosis $(53,54)$.

When combined with a low concentration of $5 \mu$ Mquinidine, WXKL at $5 \mathrm{~g} / \mathrm{l}$ inhibits Ito from epicardial and endocardial sites of coronary-perfused canine right ventricular wedge preparations in a dose-dependent manner (24). Consequently, it suppresses the substrate and trigger for VT/VF in experimental models of Brugada Syndrome $(55,56)$.

\section{Reactive oxygen species (ROS) reduction}

Oxidative stress leads to repolarization and conduction abnormalities, eventually resulting in cardiac arrhythmias (57). ROS abundance increases cardiac fibrosis and hypertrophy and reduces inotropy. Defective antioxidant mechanisms in the diabetic heart (58) are mitochondrial electron leakage, NADPH oxidase and protein kinase $\mathrm{C}$ (PKC) activation, nitric oxide synthase uncoupling (59) and a common upstream pathway involving interaction of advanced glycation end-products (AGEs) with their receptor (RAGE) to further promote ROS synthesis (60). The mitochondrial translocator protein (TSPO) links mitochondrial instability to lethal arrhythmias through ROS-induced ROS release (RIRR) (58).

Increased ROS production also activates the unfolded protein response (UPR) that accordingly reduces ion channel functionality and creates repolarization abnormalities (1). It decreases $\mathrm{Cx} 43$ protein levels in the cell and perturbs gap junction conduction, resulting in dysrhythmias and SCD. Additionally, intracellular $\mathrm{Ca}_{2}{ }^{+}$homeostasis alters and causes fibrosis (61).

WXKL at at a dose of $3 \mathrm{~g} / \mathrm{kg}$ has been shown to improve intracellular mitochondrial membrane potential and oxygen consumption in primary atrial fibroblasts by reducing ROS in diabetic rats (62). WXKL has been shown to lead to a recovery of the level of malondialdehyde (MDA), the final product of lipid peroxidation, and superoxide dismutase (SOD) in rats/rabbits subjected to I/R (63). WXKL also improves the secretions of taurine and ketone bodies to overcome I/R-induced oxidative stress (17).

\section{Gap junction modulation}

Gap junction proteins, connexins, mediate action potential propagation between cells, coordinate mechanical and electrical activity of heart muscle, and ensure simultaneous cardiac electro-mechanical activity. The age-related down-regulation of atrial $\mathrm{Cx} 43$ can facilitate the development of AF in old guinea pig hearts (64) and cause interstitial fibrosis and collagen deposition (65). In a canine model of sympathetic AF, the low expression of Cx43 was shown to be involved in AF by influencing inter-cellular channel conduction (66). Cx43 remodeling is associated with atrial denervation following epicardial GP ablation and this is ameliorated by WXKL (40) (Table I).

$\mathrm{Cx} 43$ is the primary gap junction protein that is highly expressed within mammalian ventricular muscle at the intercalated disk. A potential shift in its composition in the ventricular conduction system may contribute to VAs in patients with arrhythmogenic right ventricular cardiomyopathy (ARVC) (67). Following MI, $2.7 \mathrm{~g} / \mathrm{kg}$ WXKL has been shown to protect the ultrastructure of gap junctions within the intercalated disk, alleviate CX43 phosphorylation, increase PKC and miRNA-1 levels, and increase the VF threshold (22) (Table II).

\section{Calcium handling}

$\mathrm{Ca}^{2+}$ plays a key role in the excitation-contraction coupling and the activation of $\mathrm{Ca}^{2+}$-dependent signaling pathways. APD prolongation as a result of cardiac hypertrophy may increase the $\mathrm{Ca}^{2+}$ entry via ICaL during the long plateau phase resulting in spontaneous sarcoplasmic reticulum (SR) $\mathrm{Ca}^{2+}$ release (68).

WXKL has been shown to block ICaL reversibly in a dose-dependent manner at $5 \mathrm{~g} / 1$ in rabbit ventricular myocytes, in rats with transverse aortic constriction (TAC)-induced heart failure (19) and in an experimental model of Brugada syndrome (24), by causing shift of ICaL activation towards negative potentials (6). In the TAC rat model of heart failure, WXKL was shown to reduce ICaL by facilitating the steady-state inactivation and retarding its recovery (19). In cardiac myocytes from rats with MI, WXKL at $4 \mathrm{~g} / \mathrm{kg} /$ day was shown to significantly increase both the SR $\mathrm{Ca}^{2+}$ content and the calcium transient amplitude (69). WXKL inhibits hypoxia and the hypoxia/reoxygenation-induced increase in diastolic $\left[\mathrm{Ca}^{2+}\right]_{\mathrm{i}}$ and the decrease in 
$\Delta\left[\mathrm{Ca}^{2+}\right]_{\mathrm{i}}$. The effect of WXKL on ICaL may be attributed to its suppressive effect on $\left[\mathrm{Ca}^{2+}\right]_{i}$ transients (70). WXKL reduces the transmural dispersion of repolarization and ameliorates calcium mishandling by reducing $\left[\mathrm{Ca}^{2+}\right]_{\mathrm{i}}$ overload among Purkinje cells and in hypoxic/reoxygenated cardiomyocytes at $3 \mathrm{~g} / \mathrm{l}$ in the treatment of ventricular arrhythmias $(12,70)$. A decrease in both the expression of CaMKII and its phosphorylation at Thr-286 may be the primary mechanism through which WXKL inhibits heart failure and arrhythmia (69).

\section{CaMKII signaling}

CaMKII regulates SR Ca release and Ca reuptake (71). It is an upstream regulator of INaL in cardiac myocytes. The CaMKII-dependent phosphorylation of ICaL attenuates its inactivation, increasing the ICaL window current that plays a major role in the generation of EADs (72). Excess CaMKII activity due to HF results in enhanced sympathetic excitation and hyperphosphorylation of the ryanodine receptor (RyR), sarco/endoplasmic reticulum $\mathrm{Ca}^{2+}$-ATPase (SERCA) and phospholamban (PLB) proteins, which predisposes the cardiac myocytes to afterdepolarizations.

WXKL at $4 \mathrm{~g} / \mathrm{kg} /$ day has been shown to significantly reduce the expression of CaMKII, p-CaMKII (Thr-286) and PLB, and to increase the expression of RyR2 and FKBP12.6 in rats with MI, to inhibit arrhythmia (69). In rats with TAC, the beneficial effects of WXKL and KN93, a pharmacologic tool used to inhibit CaMKII, is attributed to the shortening of APD90 and regulation of the CaMK II signal transduction pathway, markedly decreasing the degree of collagen deposition and improving fibrosis and hypertrophy (14).

\section{Autonomic nervous system regulation}

Shortened ventricular ER potential, APD, fibrillation threshold, and triggered EAD and DAD by a high sympathetic tone increase the risk of VAs (73). In canine models, the denervated myocardium becomes hypersensitive to norepinephrine indicated by the supersensitive shortening of ERP (74). In patients with long QT syndrome (LQTS), VAs are triggered following a sympathetic burst from either emotional stress or exercise. Experimentally, sympathetic stimulation induces changes in ECG repolarization and the reduction of the fibrillation threshold (75). The ischemic and infarcted myocardium becomes a substrate sensitive to arrhythmia triggers due to the heterogeneity of the sympathetic nervous system innervation contributed by nerve sprouting (76).

Parasympathetic activation, on the other hand, is considered to be anti-arrhythmogenic (77). In rodents, $100 \%$ VF episodes were shown to be terminated with vagal nerve stimulation and the efficacy was reduced in the presence of atropine $(78,79)$. The majority of episodes of VF in patients with Brugada syndrome are observed during periods of high vagal tone, such as at rest, during sleep, or from 12 to 6 a.m. $(80,81)$.

The positive inotropic effect exerted by WXKL in right ventricular wedge preparation is likely due principally to an increase in ICaL secondary to the release of catecholamines. The boost in ICaL also contributes to restoration of the action potential dome and suppression of phase 2 reentry and pVT. WK increases ICaL via indirect adrenergic stimulation (24). 


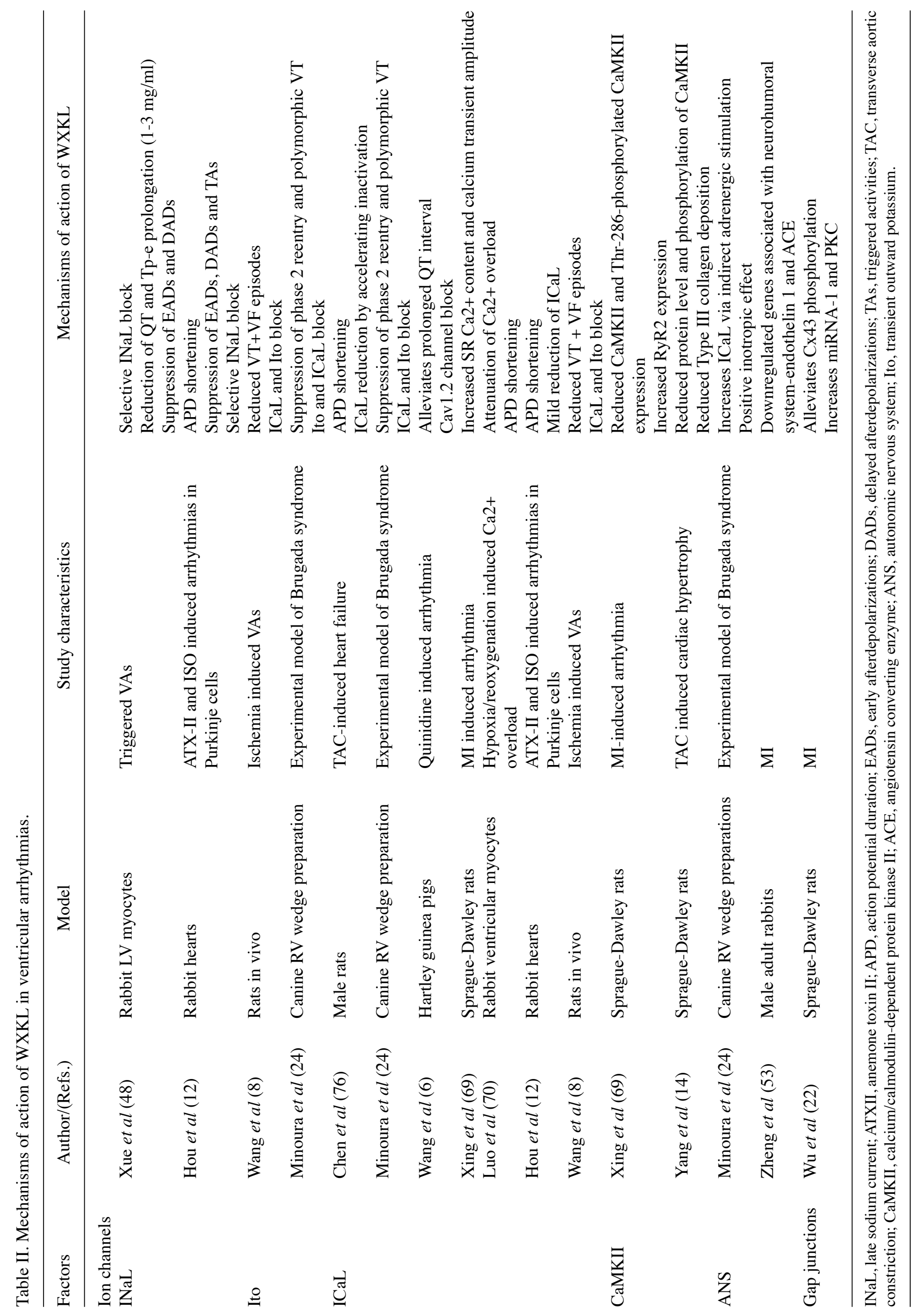


WXKL induces the release of catecholamines from sympathetic nerve endings at the tissue level exerting a tyramine-like effect (9). These ameliorative actions are accompanied by normalization of the repolarization defects in the epicardial AP and of the ECG by a diminution of the accentuated $\mathbf{J}$ waves and ST segment elevation (24). The concentration of WXKL is directly proportional to positive inotropy in wedge preparation, contradicting to that in single myocytes (24). In rabbits with MI, treatment with $817 \mathrm{mg} / \mathrm{kg} /$ day WXKL downregulates genes associated with neurohumoral system-endothelin 1 and angiotensin I converting enzyme (53).

\section{Conclusion}

Arrhythmia has multiple vulnerable facets that need to be addressed individually for optimal patient management. To date, WXKL has shown promising results in sinus rhythm reversion and maintenance, the reduction of PVCs and the suppression of EADs and DADs in a number of experimental and clinical models via diverse effects on channels chiefly INa, ICaL and Ito, CaMKII and its regulatory pathways, inflammatory mediators and $\mathrm{P}$ wave dispersion. Its efficacy in suppressing arrhythmogenesis has been compared to western drugs in several trials and the results have been satisfactory. Its safety profile as compared to severe adverse reactions and proarrhythmic tendencies of other western antiarrhythmics has further validated its promising role. With further identification of roles of gene and proteome-based therapies, second messenger systems, intercellular conduction, NCX inhibition, gating modifiers and ryanodine stabilizers, the scope of managing arrhythmias has expanded as opposed to the sole dependence of ion channel conductance-based therapies. Combining these newer approaches with actions of WXKL under diverse pathologies and circumstances could certainly fill the gaps in treating inherited as well as acquired arrhythmic syndromes.

\section{Acknowledgements}

Not applicable.

\section{Funding}

The present study was supported by grants from the National Natural Science Foundation of China (no. 81970270 to TL).

\section{Availability of data and materials}

Not applicable.

\section{Authors' contributions}

GT and TL put forward the conception and design of this review. SD, MG and SG performed the review of the literature. SD and MG contributed to the processing of the figure and tables. SD, GT and TL were major contributors to the writing and revision of the manuscript. All authors read and approved the final version of the manuscript.

\section{Ethics approval and consent to participate}

Not applicable.

\section{Patient consent for publication}

Not applicable.

\section{Competing interests}

The authors declare that they have no competing interests.

\section{References}

1. Tse G, Yan BP, Chan YW, Tian XY and Huang Y: Reactive oxygen species, endoplasmic reticulum stress and mitochondrial dysfunction: The link with cardiac arrhythmogenesis. Front Physiol 7: 313, 2016.

2. Lippi G, Sanchis-Gomar F and Cervellin G: Global epidemiology of atrial fibrillation: An increasing epidemic and public health challenge. Int J Stroke: Jan 19, 2020 (Epub ahead of print).

3. Murakoshi $\mathrm{N}$ and Aonuma K: Epidemiology of arrhythmias and sudden cardiac death in Asia. Circ J 77: 2419-2431, 2013.

4. Guo Y, Tian Y, Wang H, Si Q, Wang Y and Lip GYH: Prevalence, incidence, and lifetime risk of atrial fibrillation in China: New insights into the global burden of atrial fibrillation. Chest 147: 109-119, 2015.

5. John RM, Tedrow UB, Koplan BA, Albert CM, Epstein LM, Sweeney MO, Miller AL, Michaud GF and Stevenson WG: Ventricular arrhythmias and sudden cardiac death. Lancet 380: 1520-1529, 2012

6. Wang T, Lu M, Du Q, Yao X, Zhang P, Chen X, Xie W, Li Z, Ma Y and Zhu Y: An integrated anti-arrhythmic target network of compound Chinese medicine Wenxin Keli revealed by combined machine learning and molecular pathway analysis [corrected]. Mol Biosyst 13: 1018-1030, 2017.

7. Kalifa J and Avula UM: The Chinese herb extract Wenxin Keli: Atrial selectivity from the Far East. Hear Rhythm 9: 132-133, 2012.

8. Wang X, Wang X, Gu Y, Wang T and Huang C: Wenxin Keli attenuates ischemia-induced ventricular arrhythmias in rats: Involvement of L-type calcium and transient outward potassium currents. Mol Med Rep 7: 519-524, 2012.

9. Tang Q: Effects of Nardostachys chinensis Batal extract on sodium and calcium channels in rabbit ventricular myocytes. Chin J Cardiol 32: 267-70, 2004 (In Chinese).

10. Brenyo A and Aktas MK: Review of complementary and alternative medical treatment of arrhythmias. Am J Cardiol 113: 897-903, 2014

11. He M, Lv Z, Yang ZW, Huang JL and Liu F: Efficacy and safety of Chinese herbal medicine Wenxin Keli for ventricular premature be ats: A systematic review. Complement Ther Med 29: 181-189, 2016.

12. Hou J, Li W, Guo K, Chen XM, Chen YH, Li CY, Zhao BC, Zhao J, Wang H, Wang YP and Li YG: Antiarrhythmic effects and potential mechanism of WenXin KeLi in cardiac Purkinje cells. Hear Rhythm 13: 973-982, 2016.

13. Dong Y, Liao J, Yao K, Jiang W and Wang J: Application of traditional Chinese medicine in treatment of atrial fibrillation. Evid Based Complement Alternat Med 2017: 1381732, 2017.

14. Yang X, Chen Y, Li Y, Ren X, Xing Y and Shang H: Effects of Wenxin Keli on Cardiac hypertrophy and arrhythmia via regulation of the Calcium/Calmodulin dependent Kinase II signaling pathway. Biomed Res Int 2017: 1569235, 2017.

15. Wang X, Wang Y, Feng X, Lu Y, Zhang Y, Wang W and Zhu W: Systematic review and meta-analysis of randomized controlled trials on Wenxin Keli. Drug Des Devel Ther 10: 3725-3736, 2016.

16. Li J, Hu D, Song X, Han T, Gao Y and Xing Y: The role of biologically active ingredients from natural drug treatments for arrhythmias in different mechanisms. Biomed Res Int 2017: $4615727,2017$.

17. Jiang M, Wang Q, Chen J, Wang Y, Fan G and Zhu Y: Comparative metabonomics of Wenxin Keli and verapamil reveals differential roles of gluconeogenesis and fatty acid $\beta$-oxidation in myocardial injury protection. Sci Rep 7: 8739, 2017.

18. Burashnikov A, Petroski A, Hu D, Barajas-Martinez H and Antzelevitch C: Atrial-selective inhibition of sodium-channel current by Wenxin Keli is effective in suppressing atrial fibrillation. Hear Rhythm 9: 125-131, 2012. 
19. Chen Y, Li Y, Guo L, Chen W, Zhao M, Gao Y, Wu A, Lou L, Wang J, Liu X and Xing Y: Effects of Wenxin Keli on the action potential and L-type calcium current in rats with transverse aortic constriction-induced heart failure. Evid Based Complement Alternat Med 2013: 572078, 2013.

20. Li M, Qiu R, Tian G, Zhang X, Li C, Chen S, Zhang Q and Shang H: Wenxin Keli for Ventricular premature complexes with Heart failure: A systematic review and meta-analysis of randomized clinical trials. Complement Ther Med 33: 85-93, 2017.

21. Tse G and Yeo JM: Conduction abnormalities and ventricular arrhythmogenesis: The roles of sodium channels and gap junctions. Int J Cardiol Heart Vasc 9: 75-82, 2015.

22. Wu A, Zhao M, Lou L, Zhai J, Zhang D, Zhu H, Gao Y, Shang H and Chai L: Effect of Wenxin Granules on Gap Junction and miR-1 in rats with myocardial infarction. Biomed Res Int 2017: 3495021, 2017.

23. Du M, Huang K, Gao L, Yang L, Wang WS, Wang B, Huang K and Huang D: Nardosinone protects $\mathrm{H} 9 \mathrm{c} 2$ cardiac cells from angiotensin II-induced hypertrophy. J Huazhong Univ Sci Technolog Med Sci 33: 822-826, 2013.

24. Minoura Y, Panama BK, Nesterenko VV, Betzenhauser M, Barajas-Martínez H, Hu D, Di Diego JM and Antzelevitch C: Effect of Wenxin Keli and quinidine to suppress arrhythmogenesis in an experimental model of Brugada syndrome. Hear Rhythm 10: 1054-1062, 2013.

25. Sun J, Sun G, Meng X, Wang H, Wang M, Qin M, Ma B Luo Y, Yu Y, Chen R, et al: Ginsenoside RK3 prevents Hypoxia-Reoxygenation induced apoptosis in $\mathrm{H} 9 \mathrm{c} 2$ Cardiomyocytes via AKT and MAPK pathway. Evid Based Complement Alternat Med 2013: 690190, 2013.

26. Li L, Pan CS, Yan L, Cui YC, Liu YY, Mu HN, He K, Hu BH, Chang X, Sun K, et al: Ginsenoside Rg1 ameliorates rat myocardial ischemia-reperfusion injury by modulating energy metabolism pathways. Front Physiol 9: 78, 2018

27. Zhu D, Wu L, Li CR, Wang XW, Ma YJ, Zhong ZY, Zhao HB Cui J, Xun SF, Huang XL, et al: Ginsenoside Rg1 protects rat cardiomyocyte from hypoxia/reoxygenation oxidative injury via antioxidant and intracellular calcium homeostasis. J Cell Biochem 108: 117-124, 2009.

28. Song H, Wang P, Liu J and Wang C: Panax notoginseng preparations for unstable angina pectoris: A systematic review and meta-analysis. Phyther Res 31: 1162-1172, 2017.

29. Yu G and Wang J: Exploring mechanisms of Panax notoginseng saponins in treating coronary heart disease by integrating gene interaction network and functional enrichment analysis. Chin J Integr Med 22: 589-596, 2016.

30. Zhou Z, Wang J, Song Y, He Y, Zhang C, Liu C, Zhao H, Dun Y, Yuan D and Wang T: Panax notoginseng saponins attenuate cardiomyocyte apoptosis through mitochondrial pathway in natural aging rats. Phyther Res 32 243-250, 2018

31. Cui X, Wang S, Cao H, Guo H, Li Y, Xu F, Zheng M, Xi X and Han C: A review: The bioactivities and pharmacological applications of polygonatum sibiricum polysaccharides. Molecules 23 pii: E1170, 2018

32. Zhu X, Wu W, Chen X, Yang F, Zhang J and Hou J: Protective effects of Polygonatum sibiricum polysaccharide on acute heart failure in rats 1. Acta Cir Bras 33: 868-878, 2018.

33. Chang KS, Lee NH, Kuo WW, Hu WS, Chang MH, Tsai FJ, Tsai KH, Yang YS, Chen TS and Huang CY: Dung-Shen downregulates the synergistic apoptotic effects of angiotensin II plus Leu 27-IGF II on cardiomyoblasts. Acta Cardiol Sin 30: 56-66, 2014.

34. Tsai KH, Lee NH, Chen GY, Hu WS, Tsai CY, Chang MH, Jong GP, Kuo CH, Tzang BS, Tsai FJ, et al: Dung-Shen (Codonopsis pilosula) attenuated the cardiac-impaired insulin-like growth factor II receptor pathway on myocardial cells. Food Chem 138: 1856-1867, 2013.

35. Aguilar M and Nattel S: The past, present, and potential future of sodium channel block as an atrial fibrillation suppressing strategy. J Cardiovasc Pharmacol 66: 432-440, 2015

36. Burashnikov A, Di Diego JM, Zygmunt AC, Belardinelli L and Antzelevitch C: Atrium-selective sodium channel block as a strategy for suppression of atrial fibrillation: Differences in sodium channel inactivation between atria and ventricles and the role of ranolazine. Circulation 116: 1449-1457, 2007.

37. Burashnikov A and Antzelevitch C: Role of late sodium channel current block in the management of atrial fibrillation. Cardiovasc Drugs Ther 27: 79-89, 2013.
38. Gharaviri A, Verheule S, Eckstein J, Potse M, Krause R, Auricchio A, Kuijpers NHL and Schotten U: Effect of Na+-channel blockade on the three-dimensional substrate of atrial fibrillation in a model of Endo-Epicardial dissociation and transmural conduction. Europace 20 (Suppl 3): iii69-iii76, 2018.

39. Hu D, Barajas-Martínez H, Burashnikov A, Panama BK, Cordeiro JM and Antzelevitch C: Mechanisms underlying atrial-selective block of sodium channels by Wenxin Keli: Experimental and theoretical analysis. Int J Cardiol 207: 326-334, 2016.

40. Xiao J, Zhao Q, Kebbati AH, Deng H, Wang X, Dai Z, Yu S and Huang C: Wenxin Keli suppresses atrial substrate remodeling after epicardial ganglionic Plexi ablation. Exp Clin Cardiol 18: $153-157,2013$.

41. Zhang N, Tse G, Dahal S, Yang Y, Gong M, Chan CZY, Liu E, Xu G, Letsas KP, Korantzopoulos P, et al: Efficacy of Wenxin Keli Plus Amiodarone versus Amiodarone Monotherapy in treating recent-onset atrial fibrillation. Cardiol Res Pract 2018: 6047271,2018

42. Meng Z, Tan J, He Q, Zhu M, Li X, Zhang J, Jia Q, Wang S, Zhang $G$ and Zheng W: Wenxin Keli versus Sotalol for paroxysmal atrial fibrillation caused by hyperthyroidism: A prospective, open label, and randomized study. Evid Based Complement Alternat Med 2015: 101904, 2015.

43. Guo D, Lian J, Liu T, Cox R, Margulies KB, Kowey PR and Yan GX: Contribution of late sodium current (INa-L) to rate adaptation of ventricular repolarization and reverse use-dependence of QT-prolonging agents. Hear Rhythm 8: 762-769, 2011.

44. Antzelevitch C: Electrical heterogeneity, cardiac arrhythmias, and the sodium channel. Circ Res 87: 964-965, 2000.

45. Sicouri S, Timothy KW, Zygmunt AC, Glass A, Goodrow RJ, Belardinelli L and Antzelevitch C: Cellular basis for the electrocardiographic and arrhythmic manifestations of Timothy syndrome: Effects of ranolazine. Hear Rhythm 4: 638-647, 2007.

46. Qi D, Yang Z, Robinson VM, Li J, Gao C, Guo D, Kowey PR and Yan GX: Heterogeneous distribution of INa-L determines interregional differences in rate adaptation of repolarization. Hear Rhythm 12: 1295-1303, 2015.

47. Burashnikov A: Late INa Inhibition as an Antiarrhythmic Strategy. J Cardiovasc Pharmacol 70: 159-167, 2017.

48. Xue X, Guo D, Sun H, Wang D, Li J, Liu T, Yang L, Shu J and Yan GX: Wenxin Keli suppresses ventricular triggered arrhythmias via selective inhibition of late sodium current. Pacing Clin Electrophysiol 36: 732-740, 2013.

49. Xiao L, Koopmann TT, Ördög B, Postema PG, Verkerk AO, Iyer V, Sampson KJ, Boink GJ, Mamarbachi MA, Varro A, et al: Unique cardiac Purkinje fiber transient outward current $\beta$-subunit composition: A potential molecular link to idiopathic ventricular fibrillation. Circ Res 112: 1310-1322, 2013.

50. Li J, Xie D, Huang J, Lv F, Shi D, Liu Y, Lin L, Geng L, Wu Y, Liang D and Chen YH: Cold-inducible RNA-binding protein regulates cardiac repolarization by targeting transient outward potassium channels. Circ Res 116: 1655-1659, 2015.

51. Bohnen MS, Iyer V, Sampson KJ and Kass RS: Novel mechanism of transient outward potassium channel current regulation in the heart: Implications for cardiac electrophysiology in health and disease. Circ Res 116: 1633-1635, 2015.

52. Cho JH, Zhang R, Kilfoil PJ, Gallet R, de Couto G, Bresee C, Goldhaber JI, Marbán E and Cingolani E: Delayed repolarization underlies ventricular arrhythmias in rats with heart failure and preserved ejection fraction. Circulation 136: 2037-2050, 2017.

53. Zheng M, Liu Z, Liu N, Hou C, Pu J and Zhang S: The effect of Wenxin Keli on the mRNA expression profile of rabbits with myocardial infarction. Evid Based Complement Alternat Med 2016: 2352614, 2016.

54. Zheng R, Tian G, Zhang Q, Wu L, Xing Y and Shang H: Clinical safety and efficacy of Wenxin keli-amiodarone combination on heart failure complicated by ventricular arrhythmia: A systematic review and meta-analysis. Front Physiol 9: 487, 2018.

55. Yang G, Sau C, Lai W, Cichon J and Li W: Sleep promotes branch-specific formation of dendritic spines after learning. Science 344: 1173-1178, 2014.

56. Antzelevitch C and Patocskai B: Brugada Syndrome: Clinical, Genetic, Molecular, Cellular, and Ionic Aspects. Curr Probl Cardiol 41: 7-57, 2016

57. van Opbergen CJM, den Braven L, Delmar M and van Veen TAB: Mitochondrial Dysfunction as Substrate for Arrhythmogenic Cardiomyopathy: A search for new disease mechanisms. Front Physiol 10: 1496, 2019. 
58. Ilkan $\mathrm{Z}$ and Akar FG: The mitochondrial translocator protein and the emerging link between oxidative stress and arrhythmias in the diabetic heart. Front Physiol 9: 1518, 2018.

59. Ren X, Wang X, Yuan M, Tian C, Li H, Yang X, Li X, Li Y, Yang Y, Liu N, et al: Mechanisms and treatments of oxidative stress in atrial fibrillation. Curr Pharm Des 24: 3062-3071, 2018

60. Faria A and Persaud SJ: Cardiac oxidative stress in diabetes: Mechanisms and therapeutic potential. Pharmacol Ther 172 50-62, 2017.

61. Köhler AC, Sag CM and Maier LS: Reactive oxygen species and excitation-contraction coupling in the context of cardiac pathology. J Mol Cell Cardiol 73: 92-102, 2014.

62. Gong M, Yuan M, Meng L, Zhang Z, Tse G, Zhao Y, Zhang Y, Yuan M, Liang X, Fan G, et al: Wenxin Keli regulates mitochondrial oxidative stress and homeostasis and improves atrial remodeling in diabetic rats. Oxid Med Cell Longev 2020: 2468031, 2020.

63. Tian G, Sun Y, Liu S, Li C, Chen S, Qiu R, Zhang X, Li Y, Li M and Shang H: Therapeutic effects of Wenxin Keli in cardiovascular diseases: An experimental and mechanism overview. Front Pharmacol 9: 1005, 2018.

64. Nagibin V, Egan Benova T, Viczenczova C, Szeiffova Bacova B Dovinova I, Barancik $M$ and Tribulova N: Ageing related down-regulation of myocardial connexin-43 and up-regulation of MMP-2 may predict propensity to atrial fibrillation in experimental animals. Physiol Res 65 (Suppl 1): S91-S100, 2016.

65. Kato T, Iwasaki Y and Nattel S: Connexins and atrial fibrillation. Circulation 125: 203-206, 2011.

66. Shu C, Huang W, Zeng Z, He Y, Luo B, Liu H, Li J and Xu J: Connexin 43 is involved in the sympathetic atrial fibrillation in canine and canine atrial myocytes. Anatol J Cardiol 18: 3-9, 2017.

67. Paul M, Wichter T, Gerss J, Arps V, Schulze-Bahr E, Robenek H, Breithardt $\mathrm{G}$ and Weissen-Plenz G: Connexin expression patterns in arrhythmogenic right ventricular cardiomyopathy. Am J Cardiol 111: 1488-1495, 2013.

68. Milberg P, Fink M, Pott C, Frommeyer G, Biertz J, Osada N, Stypmann J, Mönnig G, Koopmann M, Breithardt G and Eckardt L: Blockade of I(Ca) suppresses early afterdepolarizations and reduces transmural dispersion of repolarization in a whole heart model of chronic heart failure. Br J Pharmacol 166: 557-568, 2012.

69. Xing Y, Gao Y, Chen J, Zhu H, Wu A, Yang Q, Teng F, Zhang DM, Xing Y, Gao K, et al: Wenxin-Keli regulates the calcium/calmodulin-dependent protein kinase II signal transduction pathway and inhibits cardiac arrhythmia in rats with myocardial infarction. Evid Based Complement Alternat Med 2013: 464508, 2013
70. Luo A, Liu Z, Cao Z, Hao J, Wu L, Fu C, Zeng M, Jiang W, Zhang $\mathrm{P}$, Zhao B, et al: Wenxin Keli diminishes $\mathrm{Ca}^{2+}$ overload induced by hypoxia/reoxygenation in cardiomyocytes through inhibiting $\mathrm{I}_{\mathrm{NaL}}$ and $\mathrm{I}_{\mathrm{CaL}}$. Pacing Clin Electrophysiol 40: 1412-1425, 2017.

71. Maier LS and Bers DM: Role of $\mathrm{Ca} 2+/$ calmodulin-dependent protein kinase $(\mathrm{CaMK})$ in excitation-contraction coupling in the heart. Cardiovasc Res 73: 631-640, 2007.

72. Heijman J, Voigt N, Wehrens XH and Dobrev D: Calcium dysregulation in atrial fibrillation: The role of CaMKII. Front Pharmacol 5: 30, 2014.

73. Lai Y, Yu L and Jiang H: Autonomic neuromodulation for preventing and treating ventricular arrhythmias. Front Physiol 10: 200, 2019.

74. Inoue H and Zipes DP: Results of sympathetic denervation in the canine heart: Supersensitivity that may be arrhythmogenic. Circulation 75: 877-887, 1987.

75. Yanowitz F, Preston JB and Abildskov JA: Functional distribution of right and left stellate innervation to the ventricles. Production of neurogenic electrocardiographic changes by unilateral alteration of sympathetic tone. Circ Res 18: 416-428, 1966.

76. Chen PS, Chen LS, Cao JM, Sharifi B, Karagueuzian HS and Fishbein MC: Sympathetic nerve sprouting, electrical remodeling and the mechanisms of sudden cardiac death. Cardiovasc Res 50: 409-416, 2001.

77. Ng GA: Vagal modulation of cardiac ventricular arrhythmia. Exp Physiol 99: 295-299, 2014.

78. Naggar I, Uchida S, Kamran H, Lazar J and Stewart M: Autonomic boundary conditions for ventricular fibrillation and their implications for a novel defibrillation technique. J Physiol Sci 62: 479-492, 2012

79. Meng L, Shivkumar K and Ajijola O: Autonomic regulation and ventricular arrhythmias. Curr Treat Options Cardiovasc Med 20: $38,2018$.

80. Takigawa M, Noda T, Shimizu W, Miyamoto K, Okamura H, Satomi K, Suyama K, Aihara N, Kamakura S and Kurita T: Seasonal and circadian distributions of ventricular fibrillation in patients with Brugada syndrome. Hear Rhythm 5: 1523-1527, 2008.

81. Shen MJ and Zipes DP: Role of the autonomic nervous system in modulating cardiac arrhythmias. Circ Res 114: 1004-1021, 2014.

This work is licensed under a Creative Commons Attribution-NonCommercial-NoDerivatives 4.0 International (CC BY-NC-ND 4.0) License. 